\title{
Distributed Autonomous Economic Control Strategy for Microgrid Considering Event Triggering Mechanism
}

\author{
Yulong XIONG ${ }^{\mathrm{a}}$, Shihong MIAO ${ }^{\mathrm{b}}$, Weichen $\mathrm{YANG}^{\mathrm{b}}$, Zhiwei $\mathrm{LIU}^{\mathrm{b}}$ \\ ${ }^{a}$ China-EU Institute for Clean and Renewable Energy, Huazhong University of Science \\ and Technology, Wuhan 430074, China \\ ${ }^{b}$ School of Electrical and Electronic Engineering, Huazhong University of Science and \\ Technology, Wuhan 430074, China
}

\begin{abstract}
To fully adapt to the distributed access of renewable energy, microgrid technology has been developed rapidly. Aiming at the coordination and efficient regulation of distributed resources in microgrid, this paper proposes a distributed autonomous economic control strategy for microgrid considering event triggering mechanism. First, a distributed autonomous economic control architecture is built to provide a distributed operation architecture for optimal regulation of the microgrid. Secondly, a distributed secondary control strategy based on the consensus control theory is established to realize the economic allocation of active power as well as safe and stable operation of the microgrid. On this basis, an event trigger protocol based on the consensus error of the control variables is constructed, which is conductive to reduce redundant communication. The stability of the event trigger protocol is deduced by means of Lyapunov function analysis. The simulation analysis based on the equivalent microgrid verifies that the proposed control strategy can reduce redundant communication and acquire fair distribution of reactive power and active power among DGs, realizing distributed, economical and safe operation of microgrid.
\end{abstract}

Keywords. consensus control, distributed control, autonomy economical control, event trigger

\section{Introduction}

With the continuous penetration of distributed generation (DG) such as distributed renewable energy and energy storage equipment [1], the structure and operating characteristics of the microgrid are becoming more and more complex [2], and the controllable data of the system is also showing explosive growth. In this context, the disadvantages of traditional centralized control such as low communication efficiency and poor robustness have become increasingly prominent [3]. Therefore, in order to effectively cope with the challenges brought by the large-scale access of distributed power sources, it is urgent to conduct in-depth research on microgrid control technology [4]. 
Adopting distributed control mode, distributed control strategy realizes information exchange between multiple control units through limited communication links and completes optimized control independently within each control unit [5], and then achieve the convergence and optimization of global control strategy based on consensus algorithm [6]. Compared with centralized control strategy, distributed control can realize the decomposition of communication and calculation pressure of control center, which leads to better real-time performance and robustness thus can better adapt to the plug and play of distributed control resources. It can also effectively respond to the demand for flexible adjustment of the system topology of the microgrid [7].

At present, there has been a considerable amount of research in the field of distributed control of microgrid. Olati-Saber [8] proposed a basic theoretical framework for the analysis of consensus algorithms in multi-converter networks, which lays an algorithm foundation for the research of consensus control in various fields. Hug. [9] built a distributed multi-converter system of distribution network, which aggregates controllable units such as distributed generations and electric vehicles, thereby dividing the system into multiple independent converters that operate autonomously. This study provides the basis of the operation architecture for the distributed collaborative optimization of the microgrid. Li [10] proposed an microgrid control strategy based on a hierarchical consensus algorithm. With each single power source as the control object, a three-level control model of the microgrid was established based on droop control and a consensus algorithm, which realized the distribution control of microgrid. Based on the topology of the controlled key nodes, Le et al. [11] aggregates the distributed generations and reactive power compensation devices in the microgrid into active and reactive power control groups respectively, which helps to extract the hidden loading status of each subgrid. Sun [12] proposed an event-triggered model predictive control strategy in a dynamic way, which is able to stabilize the system without the constraints of stability-related terminal. However, the consensus control strategy in researches above fail to comprehensively consider the economic characteristics of distributed control resources. Meanwhile, the continuous communication process of these researches will also cause continuous occupation and waste of communication bandwidth.

In view of the above problems, this paper proposes a distributed autonomous economical control strategy for microgrid considering event triggering mechanism. In Section II, the distributed collaborative control theory is first built to provide the basis of distributed consensus control of the microgrid. In Section III, the consensus control strategies based on traditional droop control is proposed considering the distributed, economical and stable operation of the microgrid. Section IV proposed an event triggering mechanism based on the consensus control strategy in Section III to reduce the redundant communication. Section $\mathrm{V}$ provides simulation studies to verify the effectiveness of the proposed control strategy and event triggering mechanism based on an equivalent microgrid. Section VI concludes the paper.

\section{Consensus Control Theory}

Based on the principle of consensus control, this paper formulates the local control strategy of each converter considering limited information communication and realizes the distributed autonomous control of the microgrid. Specifically speaking, each 
converter collects limited state information such as local voltage, current, active power and reactive power, and exchanges state information with neighboring converters to solve the local control variable adjustment strategy. With the iteration of "acquisitioncommunication-control-acquisition", the operation state of all converters will finally reach a consistent convergence. The state variable (or consensus variable) of the i-th converter is denoted as $\varphi_{i}$, and the state equation of the user converter is:

$$
\varphi_{i}(k+1)=\left[\sum_{j \in N_{i}} w_{i j}\left(\varphi_{j}(k)-\varphi_{i}(k)\right)+T_{i}(k)\right]+S_{i} \varphi_{i}(k)
$$

where $\mathrm{N}_{\mathrm{i}}$ is cluster of converters adjacent to the $\mathrm{i}$-th converter; $w_{i j}$ is the communication weight between the $\mathrm{i}$-th and $\mathrm{j}$-th user converter; $T_{i}$ is the track controller which can be designed according to user demand; $S_{i}$ is the self-feedback coefficient.

Based on (1), when the state information of each converter meets the following conditions, the system will achieve consistent stability.

$$
\lim _{t \rightarrow \infty}\left|\varphi_{i}(k)-\varphi_{j}(k)\right|=0
$$

The Metropolis method is adopted in this paper to calculate $w_{i j}$ :

$$
w_{i j}= \begin{cases}1 /\left(\max \left[l_{i i}, l_{j j}\right]+1\right) & j \in N_{i} \\ 1-\sum_{k \in N_{i}} w_{i k} & i=j \\ 0 & \text { other situation }\end{cases}
$$

where $l_{\text {ii }}$ represents the number of converters which have communication with the i-th converters.

\section{Distributed Autonomous Economical Control Strategy for Distribution Network}

In order to achieve the distributed autonomous economic control objectives, this paper proposes a two-layered control architecture for the user converters. The primary control adopts a droop control strategy to realize the equal proportion allocation of active power. The secondary control realizes the secondary adjustment of the system frequency, voltage and reactive power:

$$
\left\{\begin{array}{l}
f_{i}=f^{*}-k^{\mathrm{P}} I C_{i}+\gamma_{i} \\
V_{i}=V^{*}-k^{\mathrm{Q}} Q_{i}+\lambda_{i}
\end{array}\right.
$$

where $f_{i}$ and $V_{i}$ are the frequency and voltage of the i-th converter, respectively; $\gamma_{i}$ and $\lambda_{i}$ are the secondary control variables of active and reactive power control 
strategies, respectively; $k^{\mathrm{P}}$ is the droop control coefficient of incremental cost rate and frequency of microgrid; $k^{\mathrm{Q}}$ is the droop control coefficient of reactive power and voltage of microgrid.

The active power secondary control variable of the i-th converter includes the incremental cost rate secondary control variable $\left(\gamma_{i}^{\mathrm{IC}}\right)$ and frequency secondary control variable $\left(\gamma_{i}^{\mathrm{f}}\right)$ of the i-th converter's active power output:

$$
\gamma_{i}=\gamma_{i}^{\mathrm{IC}}+\gamma_{i}^{\mathrm{f}}
$$

According to the consensus control theory, the following incremental cost rate secondary control variable is constructed to realize the economical allocation of active power.

$$
\left\{\begin{array}{l}
\gamma_{i}^{\mathrm{IC}}=\left(I C_{i}^{*}(t)-I C_{i}\right)\left(k_{\mathrm{p}}^{\mathrm{IC}}+\frac{k_{\mathrm{i}}^{\mathrm{IC}}}{s}\right) \\
I C_{i}^{*}(t)=I C_{i}(t)+\left[\sum_{j \in N_{i}} w_{i j}\left(I C_{j}(t)-I C_{i}(t)\right)\right]
\end{array}\right.
$$

where $I C_{i}(t)$ is the measured value of incremental cost rate of the $i$-th converter at time $\mathrm{t} ; I C_{i}^{*}$ is the reference value of incremental cost rate of the $\mathrm{i}$-th converter updated by the consensus control protocol at time $\mathrm{t} ; k_{\mathrm{p}}^{\mathrm{IC}}$ and $k_{\mathrm{i}}^{\mathrm{IC}}$ are the proportional and integral control coefficients of the PI controller, respectively, $N_{i}$ is the cluster of converters connected to the $\mathrm{i}$-th converter.

Meanwhile, in order to meet the frequency control objectives of the microgrid, the following frequency secondary control variable with reference value is constructed.

$$
\left\{\begin{array}{l}
\gamma_{i}^{\omega}=\left(f_{i}^{*}(t)-f_{i}\right)\left(k_{\mathrm{p}}^{\omega}+\frac{k_{\mathrm{i}}^{\omega}}{s}\right) \\
f_{i}^{*}(t)=f_{i}(t)+\left[\sum_{j \in N_{\mathrm{i}}} w_{i j}\left(f_{j}(t)-f_{i}(t)\right)+b_{i}\left(f^{*}-f_{i}(t)\right)\right]
\end{array}\right.
$$

where $f_{i}(t)$ is the measured value of the i-th converter's frequency at time t; $f_{i}^{*}(t)$ is the reference value of the $\mathrm{i}$-th converter's frequency at time $\mathrm{t} ; k_{\mathrm{p}}^{\mathrm{f}}$ and $k_{\mathrm{i}}^{\mathrm{f}}$ are the proportional and integral control coefficients of the PI controller, respectively.

Likewise, the voltage secondary control term can also be divided into two parts:

$$
\lambda_{i}=\lambda_{i}^{\mathrm{V}}+\lambda_{i}^{\mathrm{Q}}
$$

In terms of voltage control, two issues need to be considered. One is to realize the tracking of the PCC node voltage to the reference voltage, and the other is to make the voltage of all user converters converge to a certain average value to prevent the system 
voltage from exceeding the safety limit. Thus, the voltage secondary control strategy is constructed as follows.

$$
\left\{\begin{array}{l}
\lambda_{i}^{\mathrm{V}}=\left(V_{i}^{*}(t)-V_{i}\right)\left(k_{\mathrm{p}}^{\mathrm{V}}+\frac{k_{\mathrm{i}}^{\mathrm{V}}}{s}\right) \\
V_{i}^{*}(t)=V_{i}(t)+\left[\varepsilon_{\text {avg }} \sum_{j \in N_{i}} w_{i j}\left(V_{j}(t)-V_{i}(t)\right)+b_{i}\left(V_{\mathrm{PCC}}^{*}-V_{\mathrm{PCC}}(t)\right)\right]
\end{array}\right.
$$

Where $V_{i}(t)$ is the measured value of the $\mathrm{i}$-th converter's voltage at time $\mathrm{t}$; $V_{i}^{*}(t)$ is the reference value of the i-th converter's voltage at time $\mathrm{t} ; \varepsilon_{\text {avg }}$ is the voltage average convergence factor, used to adjust the allowable space for voltage deviation of each converter; $k_{\mathrm{p}}^{\mathrm{V}}$ and $k_{\mathrm{i}}^{\mathrm{V}}$ are the proportional and integral control coefficients of the PI controller, respectively.

In order to meet the goal of proportional allocation of reactive power in the microgrid, the following reactive power secondary control strategy is constructed.

$$
\left\{\begin{array}{l}
\lambda_{i}^{\mathrm{Q}}=\left(Q_{i}^{*}(t)-Q_{i}\right)\left(k_{\mathrm{p}}^{\mathrm{Q}}+\frac{k_{\mathrm{i}}^{\mathrm{Q}}}{s}\right) \\
Q_{i}^{*}(t)=Q_{i}(t)+\left[\sum_{j \in N_{i}} w_{i j}\left(Q_{j}(t)-Q_{i}(t)\right)\right]
\end{array}\right.
$$

where $Q_{i}(t)$ is the measured value of the i-th user converter's reactive power output at time $\mathrm{t} ; Q_{i}^{*}(t)$ is the reference value of the $\mathrm{i}$-th converter's reactive power output at time $\mathrm{t} ;{ }^{\mathrm{Q}}{ }^{\mathrm{Q}}$ and $k_{\mathrm{i}}^{\mathrm{Q}}$ are the proportional and integral control coefficients of the PI controller, respectively.

\section{Event Triggering Mechanism}

In order to realize effective communication within the microgrid and save microgrid communication and computing resources, the event trigger mechanism is introduced. Figure 1 and Figure 2 show the information communication architecture and process of event triggering mechanism in the microgrid. represents the disturbance to $\mathrm{i}$-th user converter.

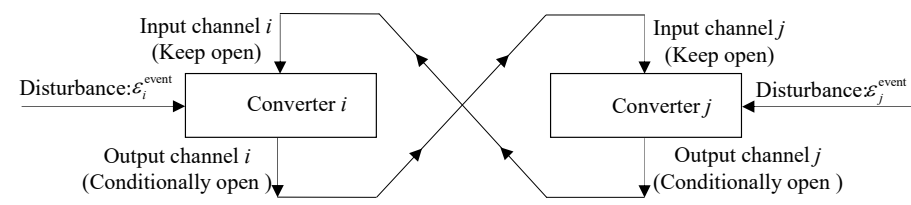

Figure 1. Information communication architecture diagram based on event triggering mechanism. 


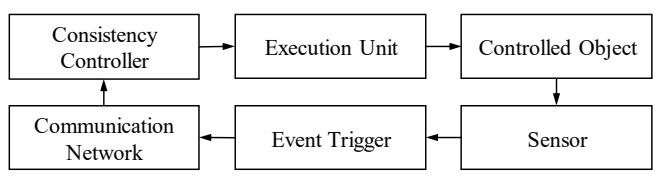

Figure 2: Event triggering process diagram.

This paper constructs the event trigger function of the distributed autonomous control of the microgrid as follows:

$$
\left\|e_{x_{i}}(t)\right\|_{2}^{2}>\eta_{i}\left\|\sum_{j \in N_{i}} a_{i j}\left(x_{i}\left(t_{t r i, i}\right)-x_{j}\left(t_{t r i, j}\right)\right)\right\|_{2}^{2}
$$

In (11), $e_{x_{i}}(t)=x_{i}(t)-x_{i}\left(t_{t r i, i}\right) . x_{i}(t)$ is the state variables of the $\mathrm{i}$-th converter. In this paper, $x_{i}(t)$ includes $I C_{i}(t), \omega_{i}(t), V_{i}(t)$ and $Q_{i}(t) . e_{x_{i}}(t)$ is the measurement deviation of $x_{i}(t)$ at time t. $t_{t r i, i}$ is the latest event trigger time. $\eta_{i}$ is the proportional control coefficient.

Studying the event trigger function shown in (11), it can be told that the left term of the inequality represents the deviation of the current operating state of the i-th converter from the operating state of the latest trigger time. Meanwhile, the term on the right side of the inequality represents the consistency deviation between the i-th converter and the adjacent $\mathrm{j}$-th converter at the time of the latest event triggering. If the i-th converter cannot maintain the stable state of the previous event trigger when disturbance occurs, the measurement deviation $e_{x_{i}}(t)$ will increase. When $e_{x_{i}}(t)$ exceeds the given threshold, which is $\eta_{i}\left\|\sum_{j \in N_{i}} a_{i j}\left(x_{i}\left(t_{t r i, i}\right)-x_{j}\left(t_{t r i, j}\right)\right)\right\|_{2}^{2}$, the $\mathrm{i}$-th converter will not be able to maintain stable operation based only on the system operation information obtained when the last event trigger, and system consistency will be destroyed. At this time, the information communication channel between the i-th converter and its neighbor converters needs to be opened to maintain the consistency and stability of the system.

Based on the given control strategy in Section III. B, the dynamic characteristics of the system can be expressed as (12) and (13).

$$
\begin{gathered}
\dot{X}(t)=-c L \hat{X}(t)=-c L(X(t)+E(t)) \\
\left\{\begin{array}{l}
X(t)=\left[x_{1}(t), x_{2}(t), \cdots, x_{N}(t)\right]^{T} \\
\hat{X}(t)=\left[\hat{x}_{1}(t), \hat{x}_{2}(t), \cdots, \hat{x}_{N}(t)\right]^{T} \\
E(t)=\left[e_{1}(t), e_{2}(t), \cdots, e_{N}(t)\right]^{T} \\
\hat{x}_{i}(t)=x_{i}\left(t_{k, i}\right)
\end{array}\right.
\end{gathered}
$$

where $\mathrm{L}$ represents the Laplace matrix of the system topology diagram; $\mathrm{c}$ is the control constant. 
To verify the stability of the proposed event trigger function, a Lyapunov function is constructed as follows:

$$
V(t)=\frac{1}{2} X^{T}(t) X(t)
$$

Bringing (12) into (14):

$\dot{V}(t)=-c X^{T}(t) L \hat{X}(t)=-c\left(\hat{X}^{T}(t)-E^{T}(t)\right) L \hat{X}(t)=-c \hat{X}^{T}(t) L \hat{X}(t)+c E^{T}(t) L \hat{X}(t)$

Noting that:

$$
E^{T}(t) L \hat{X}(t) \leq \frac{1}{2} E^{T}(t) L E(t)+\frac{1}{2} \hat{X}^{T}(t) L \hat{X}(t)
$$

Equation (17) can be transformed into:

$$
\begin{aligned}
\dot{V}(t) & \leq-c \hat{X}^{T}(t) L \hat{X}(t)+\frac{c}{2}\left(E^{T}(t) L E(t)+\hat{X}^{T}(t) L \hat{X}(t)\right) \\
& =-\frac{c}{2} \hat{X}^{T}(t) L \hat{X}(t)+\frac{c}{2} E^{T}(t) L E(t)
\end{aligned}
$$

Bringing (12) into (17):

$$
\dot{V}(t) \leq-\frac{c}{2}\left(1-\lambda_{n}^{2} \eta_{\max }\right) \hat{X}^{T}(t) L \hat{X}(t)
$$

where $\eta_{\max }=\max \left\{\eta_{i}, i=1, \cdots, N\right\}$ and $\lambda_{n}$ is the maximum eigenvalue of the Laplace matrix L. According to (18), it can be told that when control coefficient $\mathrm{c}$ and max meet the condition that $\mathrm{c}>0$ and $0<\eta_{\max }<\frac{1}{\lambda_{n}^{2}}, \dot{V}(t) \leq 0$. Under this condition, formulating system communication and control strategies based on the event-triggered function shown in (11) will ensure the consistency and stability referring to LaSalle invariance principle.

\section{Simulation Analysis}

In this section, the case studies based on a test microgrid system are carried out. The simulation results of load changes and converter switching are shown and analyzed, which verifies the effectiveness of the proposed control strategy and event trigger mechanism. All simulations are carried out via PSCAD/EMTDC. 


\subsection{Simulation System}

In order to verify the effectiveness of the control strategy proposed in this paper, a simulation model of the AC microgrid as shown in Figure 3 was built. The microgrid is constructed by 3 converters those are coupled by several lines. Converter 1 and Converter 2 are connected to the superior level grid node through public connection bus (i.e. the PCC bus), while Converter 3 is coupled with Converter 2 and has weak connection to the superior node. The incremental cost rate of each converter is simplified to $I C_{i}=2 \alpha_{i} P_{i}+\beta_{i}$. The specific value of the cost parameters of the converters are shown in Table 1. Parameters of the coupling lines among different converters are shown in Table 2. Main parameters such as PI coefficients are shown in Table 3. The rated voltage of the system is $0.38(\mathrm{kV})$, while the rated frequency is set to be $50(\mathrm{~Hz})$. The public connection bus of the system is equipped with loads, of which the active load is resistive load and the reactive load is inductive load. The initial load of the system is $80+\mathrm{j} 30(\mathrm{kV} \bullet \mathrm{A})$. The voltage control goal of the system is to realize the tracking of the rated voltage of the PCC bus. The frequency control goals of the converters are to restore the frequencies of themselves to $50 \mathrm{~Hz}$.

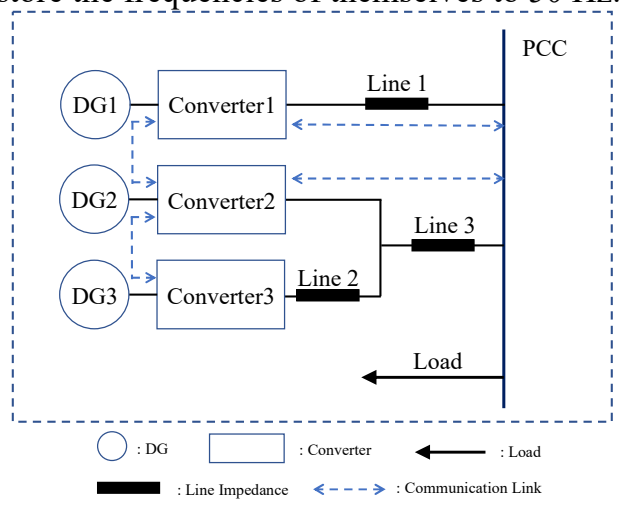

Figure 3. Structure diagram of the simulation system.

Table 1. Cost factors of different DGs.

\begin{tabular}{ccc}
\hline DG & $\left(\$ \cdot \mathrm{kW}^{-2}\right)$ & $\left(\$ \cdot \mathrm{kW}^{-1}\right)$ \\
\hline DG1 & 0.13 & 0.059 \\
DG2 & 0.095 & 0.0085 \\
DG3 & 0.082 & 0.0402 \\
\hline
\end{tabular}

Table 2. Parameters of coupling lines.

\begin{tabular}{cc}
\hline Line & Impedance $(\Omega)$ \\
\hline Line1 & $0.15+\mathrm{j} 0.2$ \\
Line2 & $0.04+\mathrm{j} 0.1$ \\
Line3 & $0.05+\mathrm{j} 0.075$ \\
\hline
\end{tabular}

Table 3. Main control parameters.

\begin{tabular}{cc}
\hline Symbol & value \\
\hline$k^{\mathrm{P}}$ & 0.01 \\
$k^{\mathrm{Q}}$ & 0.001 \\
$C_{\mathrm{avg}}$ & 0.2 \\
$k_{\mathrm{p}}^{\omega} / k_{\mathrm{i}}^{\omega}$ & $0.5 / 20$ \\
$k_{\mathrm{p}}^{U} / k_{\mathrm{i}}^{U}$ & $0.1 / 20$ \\
$k_{\mathrm{p}}^{Q} / k_{\mathrm{i}}^{Q}$ & $0.001 / 1$ \\
\hline
\end{tabular}




\subsection{Simulation Results}

In order to verify the effectiveness of the discrete and consistent distributed economical control strategy and event trigger mechanism proposed in this paper, this paper considers the microgrid load changes and DG switching, and simulates and analyzes the built microgrid system.

\subsubsection{Simulation Results of Load Change}

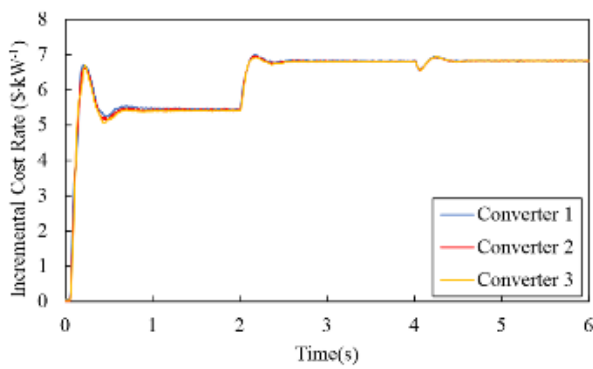

(a) Incremental cost of converters

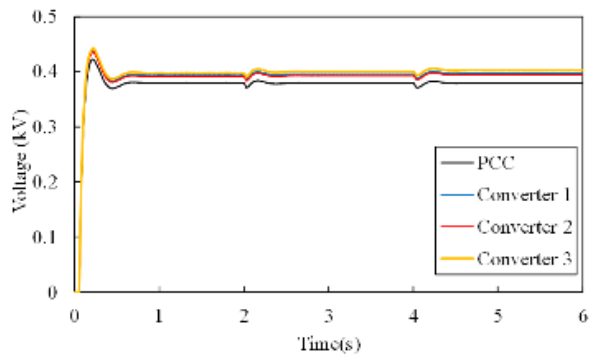

(c) Voltage of PCC/Converters

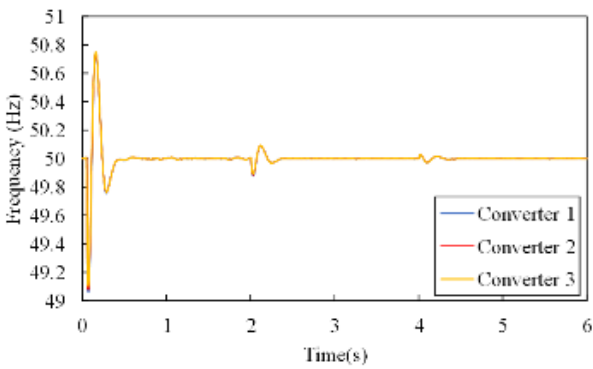

(b) Frequency of converters

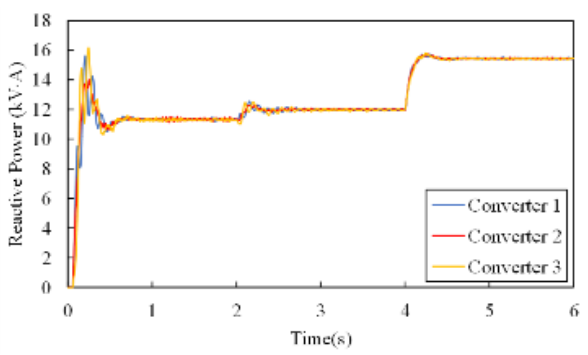

(d) Reactive power output 

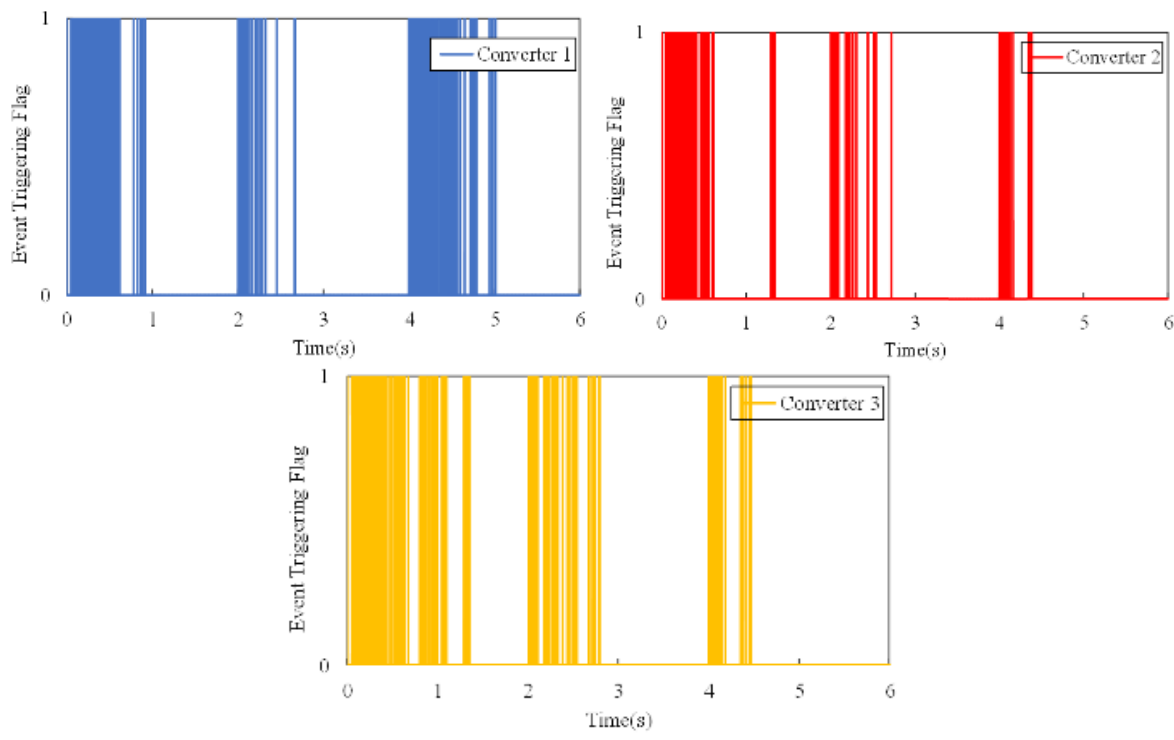

(e) Event trigger condition of Converters

Figure 4. Simulation results of system load change.

Figure 4 shows the simulation results in the case of system load changes. The initial active load of the system is $80 \mathrm{~kW}$ and the initial reactive load is $30 \mathrm{kVar}$, respectively. At $2 \mathrm{~s}$, the active load is increased to $100 \mathrm{~kW}$. Furthermore, the reactive load is increased to $40 \mathrm{kVar}$ at $4 \mathrm{~s}$.

It can be seen from Figure 4(a)-(d) that based on the consensus control strategy proposed in this paper, the distributed autonomous economic control of the distribution network user agent can be effectively realized.

Specifically speaking, when the system starts from the shutdown state, it can reach the stable operating state after a $0.5 \mathrm{~s}$ transient process. During the transient process, the control variables did not experience obvious oscillations. Although the voltage of the PCC node and the frequency of each user agent experienced one and three times of overshoot, respectively, the overshoots are relatively small and the system quickly returned to the normal operating range after overshooting. After entering the steady operation state, the incremental cost rate of all user agents are consistent, which effectively realized the economic allocation of active power among the agents (refer to Figure 5.(a)). The operating frequency of each user agent is consistent with the frequency of the superior grid, which maintains at $50 \mathrm{~Hz}$ (refer to Figure 5.(b)). Meanwhile, refer to Figure 5.(c), the voltage of PCC is maintained at $0.38 \mathrm{kV}$ and the voltage of each user agent is lower than 1.05 p.u. of the rated voltage while the voltage gaps among different user agents are small, too. Besides, the reactive power outputs of 3 user agents are basically the same, which realizes the equal allocation of reactive power among the agents. After the distribution network encounters active and reactive load changes in $2 \mathrm{~s}$ and $4 \mathrm{~s}$, the system resumes consistent and stable operation after a short and smooth transient process. The increase of the active and reactive load of the system is shared by each user agent in proportion. Thus, the control strategy proposed in this paper can effectively realize the designed distributed control goal under power fluctuation scenarios, which is conducive to the realization of stable, distributed and economical control of the distribution network. After the microgrid encounters active 
and reactive load changes in $2 \mathrm{~s}$ and $4 \mathrm{~s}$, the system resumes consistent and stable operation after a short and smooth transient process. The increase of the active and reactive load of the system is shared by each converter in proportion. Thus, the control strategy proposed in this paper can effectively realize the designed distributed control goal under power fluctuation scenarios, which is conducive to the realization of stable, distributed and economical control of the microgrid.

According to Figure 4.(e), during the startup and power fluctuation transient process, the event trigger flag of each converter is turned on and remains triggered during system transients. After the system reaches a steady state, the event trigger flag of each converter is gradually turned off. In the whole $6 \mathrm{~s}$ simulation time, the output channel opening time of Converter 1,2 and 3 is $22.50 \%, 19.83 \%$, and $28 \%$ respectively. It can be seen that the proposed event trigger mechanism can accurately trigger the information output of the converter when the system experience power fluctuation, and effectively reduces unnecessary information communication thus saves the communication resources of the system.

\subsubsection{Simulation Results of Converter Switching}

To verify the adaptability of the proposed control strategy and event trigger mechanism to plug and play applications, the simulations of converter switching are carried out. As shown in Figure 3, Converter 3 disconnects from the system at $2 \mathrm{~s}$, and reconnects to the system at $4 \mathrm{~s}$. In the process of converter switching, the system communication weight matrix is updated based on (4).

It can be seen from Figure 5(a)-(d) that, after Converter 3 exits the operation, the active and reactive power output of Converter 1 and Converter 2 rises rapidly, satisfying the load demand as well as restoring the voltage at PCC and operating frequency to the reference level. In the transient and steady state of the system, adjusted by the active and reactive power secondary control variables, the active and reactive power output of Converter 1 and Converter 2 can maintain the operation principle of equal incremental cost rate allocation of active power and equal proportion allocation of reactive power, thus fulfill the economical operation requirements of the system. After Converter 3 is put back into operation again, the incremental cost rate and reactive power output of each user converge after oscillation, and the system frequency and voltage of PCC can also be restored to the reference value. Therefore, the proposed control strategy can fully adapt to the plug-and-play application requirements of the microgrid, and realize the distributed autonomous economical control of the microgrid.

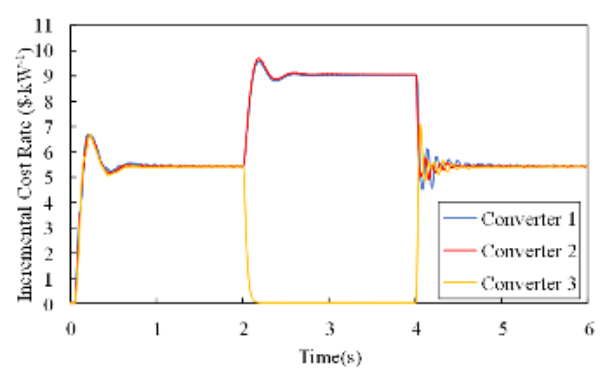

(a) Incremental cost of Converters

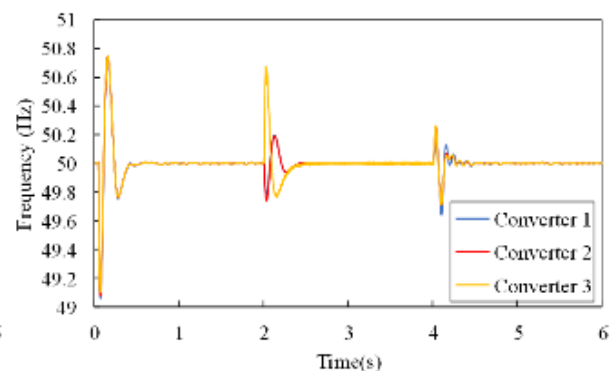

(b) Frequency of Converters 


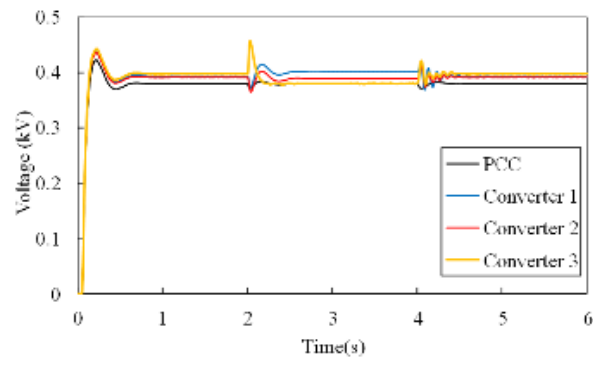

(c) Voltage of PCC/Converters
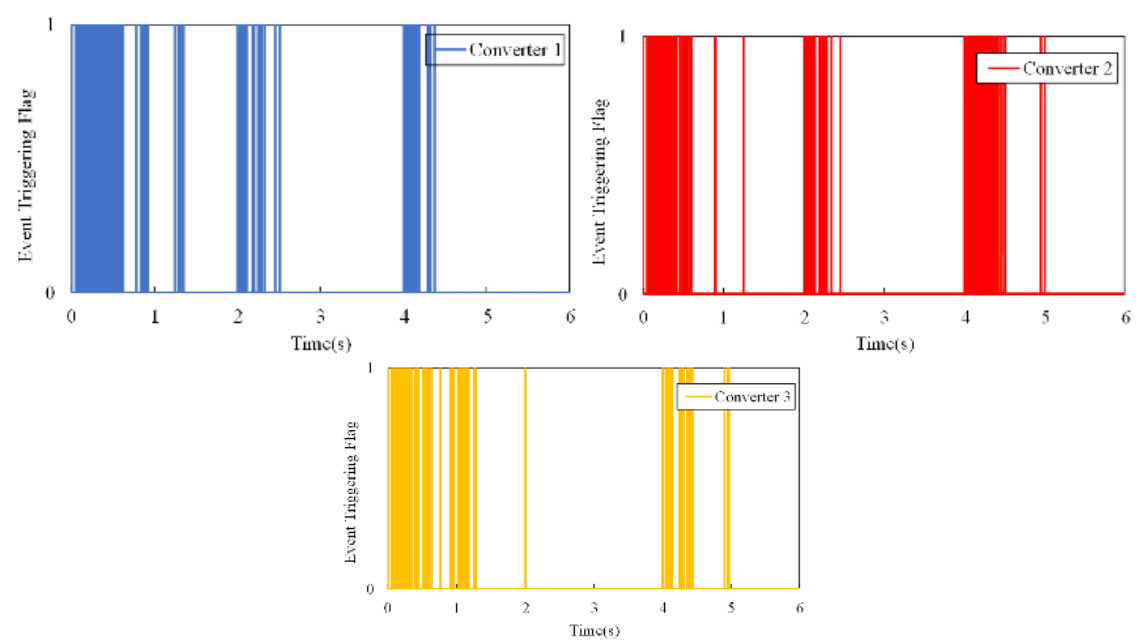

(e) Event trigger condition of Converters

Figure 5. Simulation results of converter switching.

As shown in Figure 5(e), it is the information communication situation of the system before and after the converter 3 is cut out and put in. It can be seen that Converter 3 no longer participates in information communication after switching out. Starting from the moment Converter 3 is cut out, event trigger flags are Converter 1 and Converter 2 are turned on and remain triggered before the system reaches a steady state. After Converter 1 and Converter 2 achieve consistency and stability, the event trigger flags are turned off, while Converter 1 and Converter 2 maintain stable operation based on the latest communication information. Then, after Converter 3 is reconnected to the system, communication is triggered again among the adjacent user converters and the event trigger flags of all converters are turned on. After the system re-enters the steady state, the communication is closed accordingly. The event trigger flags of all converters are turned off and all converters remain uniformly stable operation based on the status information of adjacent converters obtained at the last trigger. Therefore, the event trigger principle based on the consistency deviation proposed in this paper is fully adapted to the "plug and play" application situations. 
An example of table styling. It is recommended to add cross references to tables, i.e., please, check Table 1. The style should be switched to Normal.

\section{Conclusion}

This paper proposes a distributed autonomous economic control strategy for distribution networks that considers event triggering. Regarding the coordinated control of the distribution network, the distributed consensus control strategy is proposed aiming at the economical allocation of active power, the equal proportional allocation of reactive power and the tracking of system frequency and voltage of PCC to the referenced value. On this basis, an event trigger mechanism based on the consistency deviation of control variables is established, which effectively realizes the necessary and efficient communication between adjacent user agents of the distribution network. The simulation studies verify the effectiveness of the effectiveness of the proposed control strategy and event triggering mechanism under different operation situations. Several conclusions can be drawn as follows.

1) The distributed economic control strategy of the distribution network proposed in this paper can effectively maintain the track of system frequency and voltage of PCC to the reference value. It can also realize the economic allocation of active power and proportional allocation of reactive power among user agents. Its good dynamic performance for different scenarios such as power fluctuations and agents switching makes it possess superior practicability.

2) The event triggering mechanism can effectively judge whether the disturbance will lead to changes in the operating state of the system, thereby ensuring the realization of necessary communication and effectively avoiding redundant information communication. Based on the proposed event triggering mechanism, system communication and computing resources can be significantly saved.

3) Applying the distributed autonomous economic control strategy proposed in this article, the solution of the control strategy and the communication of state information can be decomposed to each user agent. Then, under the condition of limited information communication, the proposed control strategy can effectively alleviate the shortcomings of high communication requirements and central information processing pressure of traditional centralized control strategies, thereby promoting the distributed autonomous economic operation of the distribution network.

Since DC/AC hybrid microgrid can better utilize the distributed renewable generation resource, next step of research could be the economical control strategy of DC/AC hybrid microgrid.

\section{References}

[1] H Qu, X Li, L Yang, Y Huang, M Wang, et al. Multi-objective distribution network dynamic reconfiguration and DG control considering time variation of load and DG [J]. High Voltage Engineering, 2019, 45(3):873-881.

[2] S Li, B Zhang, L Peng, T Mei, X Li, et al. Dynamic island partitioning strategy for active distribution network considering adjustment ability of flexible load [J]. High Voltage Engineering, 2020, 45 (6): $1835-1842$.

[3] Q Xu, J Xiao, P Wang, et al. A decentralized control strategy for economic operation of autonomous AC, DC, and Hybrid AC/DC Microgrids [J]. IEEE Transactions on Energy Conversion, 2017, 32(4):13451355. 
[4] Z Y Lv, Z J Wu, X B Dou, et al. A distributed droop control scheme for islanded DC microgrid considering operation costs [J]. Proceedings of the CSEE, 2016, 36(4): 900-910.

[5] H Sun, Z Shen, W Zhou, Q Sun, M Sun, et al. Multi-objective congestion dispatch of active distribution network based on source-load coordination [J]. Automation of Electric Power Systems, 2017, 41(16):88-95.

[6] H Xin, D Gan, C Dai, H Li, N Li. Virtual power plant-based distributed control strategy for multiple distributed generators [ J]. IET Control Theory and Applications, 2013, 7:90-98.

[7] N Moshtagh, M Michael, A Jadbabaie, K Daniilidis. Vision-based, distributed control laws for motion coordination of nonholonomic robots [J]. IEEE Transactions on Robotics, 2009, 25(4):851-860.

[8] R Olati-Saber, J A Fax, R M Murray. Consensus and cooperation in networked multi-agent systems [J], Proceedings of the IEEE, 2007, 95(1):215-233.

[9] Hug, G., Kar, S., and Wu, C. Consensus + innovations approach for distributed multiagent coordination in a microgrid. IEEE Transactions on Smart Grid, 2017, 6(4):1893-1903.

[10] W Li, C Zhou, R Ye, J Wu. Research on active distribution network control strategy based on hierarchical consistency algorithm [J]. Electric Power Information and Communication Technology, 2016, 14(5):69-75.

[11] P Lin, C Jin, J Xiao, et al. A Distributed control architecture for global system economic operation in autonomous hybrid AC/DC microgrids [J]. IEEE Transactions on Smart Grid, 2018.

[12] Z Q Sun, C Li, J H Zhang, Y Q Xia. Dynamic event-triggered MPC with shrinking prediction horizon and without terminal constraint [J]. IEEE Transactions on Cybernetics, 2021 (Early Access). 